\title{
Measuring the Recovery Orientation of Assertive Community Treatment
}

\author{
Michelle P. Salyers, PhD \\ Associate Professor, Department of Psychology, Indiana University Purdue University Indianapolis \\ (IUPUI); Co-Director, ACT Center of Indiana; Research Scientist, Regenstrief Institute, Inc. \\ Laura G. Stull, PhD \\ Assistant Professor, Anderson University \\ Angela L. Rollins, PhD \\ Research Scientist, VA HSR\&D Center on Implementing Evidence-based Practice, Roudebush VAMC; \\ Research Director, ACT Center of Indiana; Assistant Research Professor, Department of Psychology, \\ IUPUI
}

John H. McGrew, PhD

Professor, Department of Psychology, IUPUI

Lia J. Hicks, MBA

Associate Director, Innovative Practices, Consultation \& Training, Adult \& Child Center/ACT Center of Indiana

Dave Thomas, BS

Peer Recovery Specialist, Adult \& Child Center

Doug Strieter, LCSW

Associate Director, Adults Services Line, Adult \& Child Center

Running Head: Recovery Oriented ACT 
Acknowledgements: This study was funded by an IP-RISP grant from the National Institute of Mental Health (R24 MH074670; Recovery Oriented Assertive Community Treatment). We appreciate the involvement of staff and consumers who participated, and the assistance of Angela Donovan, Candice Hudson, Dawn Shimp, and Jenny Lydick in the preparation of this manuscript.

Address correspondence to:

Michelle P. Salyers, Ph.D.

Department of Psychology, IUPUI

402 N. Blackford Street

Indianapolis, IN 46202

(317) 274-2904 (tel.); (317) 988-3222 (FAX); Email: MPSALYER@IUPUI.EDU

Accepted version; Final version published as Salyers, M. P., Stull, L. G., Rollins, A. L., McGrew, J. H., Hicks, L.J., Thomas, D., \& Strieter, D. (2013). Measuring the Recovery Orientation of Assertive Community Treatment. Journal of the American Psychiatric Nurses Association, 19, 117-128. 


\begin{abstract}
Background: Approaches to measuring recovery orientation are needed, particularly for programs that may struggle with implementing recovery-oriented treatment. Objective: A mixed methods comparative study was conducted to explore effective approaches to measuring recovery orientation of Assertive Community Treatment (ACT) teams. Design: Two ACT teams exhibiting high and low recovery orientation were compared using surveys, treatment plan ratings, diaries of treatment visits, and teamleader-reported treatment control mechanisms. Results: The recovery-oriented team differed on one survey measure (higher expectations for consumer recovery), treatment planning (greater consumer involvement and goal-directed content), and use of control mechanisms (less use of representative payee, agency-held lease, daily medication delivery, and family involvement). Staff and consumer diaries showed the most consistent differences (e.g., conveying hope and choice) and were the least susceptible to observer bias, but had the lowest response rates. Conclusions: Several practices differentiate recovery orientation on ACT teams, and a mixed-methods assessment approach is feasible.
\end{abstract}


Recovery is at the forefront of mental health consumer concerns (Deegan, 1988; Mead \& Copeland, 2000; Ridgway, 2000) and should be the guiding vision of services according to the President's New Freedom Commission (President's New Freedom Commission on Mental Health, 2003) and mental health advocates (Anthony, 2000, 2004). Recovery oriented care is still a high priority for service systems (SAMHSA, 2011). However, at times, there can be a tension between providing recovery-oriented services and empirically validated treatments (Anthony, Rogers, \& Farkas, 2003); one key exemplar of this tension is Assertive Community Treatment (ACT). ACT is recognized as an evidence-based practice that is successful in engaging consumers with severe mental illness, reducing hospitalization, increasing housing stability, and reducing homelessness (Bond, Drake, Mueser, \& Latimer, 2001; Coldwell \& Bender, 2007; Phillips et al., 2001). Although ACT is a highly effective mode of delivering services and is widely implemented, some observers have questioned its recovery orientation (Anthony et al., 2003; Gomory, 2001; Williamson, 2002).

A recent consensus-based definition of recovery is "a process of change through which individuals improve their health and wellness, live a self-directed life, and strive to reach their full potential" (SAMHSA, 2011). Other critical elements of a recovery orientation include a focus on health, home, having a sense of purpose, and community. In this light, the ACT model has many structural aspects that support recovery. ACT is a holistic approach, that can serve as a platform for integrating primary and behavioral health care (Weinstein, Henwood, Cody, Jordan, \& Lelar, 2011) and includes nursing and medical staff working side by side with rehabilitation professionals. ACT programs have been very successful in helping people with severe mental illness increase time spent in the community and find and maintain stable housing (Coldwell \& Bender, 2007; Mueser, Bond, Drake, \& Resnick, 1998). Although ACT programs have been less successful in employment and social domains, some studies show benefits in these areas as well (Becker, 1999; Mueser et al., 1998). 
Although ACT can be recovery oriented, practicing in a recovery-oriented fashion may be particularly challenging for ACT teams, given that admission criteria ensure that many consumers will be "treatment noncompliant" (Angell, Mahoney, \& Martinez, 2006; Salyers \& Tsemberis, 2007). In addition, ACT teams use assertive strategies that include repeated engagement attempts, close medication monitoring, behavioral contracting, outpatient commitments, and representative payeeships. Because these approaches to engagement and treatment are commonly used, it has been suggested that ACT programs must employ some form of coercion in order to be effective (Dennis \& Monahan, 1996).

The extent to which ACT teams are recovery oriented is unclear. ACT staff report primarily using "friendly persuasion," and only rarely using coercive methods, in facilitating behavior change, (Neale \& Rosenheck, 2000). Consumers also report infrequent use of coercion by ACT teams (Tschopp, Berven, \& Chan, 2011). Although staff and consumer perceptions may be subject to a social desirability bias, most consumers also report being highly satisfied with ACT services (Rapp \& Goscha, 2004). Even in the absence of coercion, who controls key treatment choices also relates to recovery oriented care. Moser and Bond (2009) found wide variability in the degree to which ACT teams control aspects of consumers' lives (e.g., involuntary commitment, depot injections, representative payee, agency supervised housing).

Given the potential discrepancies between ACT and recovery-oriented care, we were interested in developing a more comprehensive method to assess recovery at a site level and then, using this data, help to describe and understand differences in recovery oriented practices across ACT exemplars. To date, recovery orientation of programs has been assessed primarily through surveys (Bedregal, O'Connell, \& Davidson, 2006; O'Connell, Tondora, Evans, Croog, \& Davidson, 2005; Salyers, Tsai, \& Stultz, 2007). One recent study specifically examined recovery orientation in ACT (Kidd et al., 2010) and found moderate levels of recovery orientation based on perceptions of consumers, providers, and family members. Although survey approaches may be helpful, surveys can also pull for positive response bias 
(e.g., belief in recovery, consumer choice, absence of coercion). On the other hand, other methods of assessment may be too labor intensive or intrusive to be practical. Thus, while we incorporated a broader measurement approach to provide a more accurate and deeper picture of recovery orientation, we were also interested in assessing the utility and feasibility of different assessment methods. We present findings from a multi-method approach, combining survey data with chart reviews and observer ratings, to develop more systematic methods to assess the recovery orientation of ACT teams. Based on key informant ratings, we identified extreme programs (high and low on recovery orientation) and applied quantitative and qualitative methods to determine how the programs differed. This manuscript focuses on the quantitative findings; qualitative findings are described in another manuscript [Author Cite]. We expected the high recovery-oriented ACT team to have more satisfied, hopeful consumers, who were managing their illnesses more independently and participating in meaningful activities. We also expected staff-rated consumer optimism to differ across teams. In addition to surveys, we expected recovery orientation to be distinguished through observer ratings of treatment plans, and staff and consumer ratings of visit activities.

\section{Methods}

\section{Participants}

Participants included staff and consumers on two ACT teams (see below for site selection measures). Team A had 9 staff and 43 consumers, and Team B had 12 staff and 74 consumers. Sites were selected from certified ACT teams in [state]. ACT programs followed standards established by the state's Division of Mental Health and Addiction to maintain certification and funding. Staffing includes a master's level team leader, a psychiatrist, at least one registered nurse, vocational specialist, and substance abuse specialist, and other case managers. Standards specified frequency, intensity, and type of services offered (e.g., symptom management, crisis assessment and intervention) to ensure 
consistency across teams. Because ACT teams are designed to serve consumers with severe mental illness (SMI) who have the most severe disabilities, state certification require that $80 \%$ of $\mathrm{ACT}$ consumers have a DSM-IV diagnosis on Axis I of 295-296 (schizophrenia, bipolar disorder, and other major mood disorders). In addition, ACT admission criteria include at least one of the following: extended or frequent hospitalizations or use of emergency services, persistent symptoms, co-occurring substance use, criminal justice involvement, or homelessness. All ACT teams had a consultant/trainer who supported implementation through training and program monitoring including annual on-site fidelity visits, other visits as requested, and frequent email and phone contact with team leaders.

\section{Measures}

\section{Site Selection - Fidelity to ACT}

At the time of the study, all state ACT teams were assessed annually for fidelity to the ACT model using the 28-item Dartmouth Assertive Community Treatment (DACTS; (Teague, Bond, \& Drake, 1998). Each item is rated on a 5-point behaviorally anchored scale, ranging from 1 ("not implemented") to 5 ("fully implemented"). The DACTS discriminates types of programs (Teague et al., 1998) and is sensitive to change over time in implementation efforts (McHugo et al., 2007). Inter-rater reliability of the DACTS was .99 in the National Implementing Evidence-Based Practices Project (McHugo et al., 2007). DACTS ratings were completed by ACT consultant/trainers during regularly scheduled visits to the programs and were based on observation of the team meeting, interviews with staff and consumers, and chart reviews. Teams were required to have scored at or above a 4.0 on their most recent DACTS assessment to be eligible.

Site Selection - Recovery Exemplars 
Four consultant/trainers rated all certified ACT teams in the state on the degree to which staff instill hope, foster personal responsibility for illness management, and help consumers pursue meaningful life activities (each of the three items was on a scale from 1 to 5 , with 5 indicating the highest level of recovery orientation). These items were selected based on emerging themes that characterize recovery, including hope, personal responsibility, and meaningful lives (Noordsy et al., 2002). A mean score was derived for each team. Inter-rater reliability of the recovery orientation score was assessed for teams with more than one rater $(n=12)$. The intraclass correlation was adequate (ICC $=.70$ overall). In addition, we did a second round of ratings three months later. The pearson correlation between ratings was $r=.70, p<.001$, indicating adequate stability across time. At the second round of ratings, there were 15 teams with more than one rater, and agreement between raters was good (ICC = .86 overall).

Our intent was to recruit the two teams rated by consultant/trainers as highest in recovery orientation and the two teams rated lowest. Out of the 32 certified ACT teams at the time of the study, the two highest rated teams accepted participation and were included. Among the four lowest rated teams, one was recruited, one declined participation, and two did not meet the minimum fidelity threshold. The fifth lowest rated team was selected and assessed. However, because the fifth-lowestrated team was not substantially different than several teams rated closely above it, we compared just the highest (Team A) and lowest (Team B) rated teams to remain closer to our intent to compare extremes. Mean informant recovery orientation scores across the two rating periods were 4.75 for Team A and 2.35 for Team B.

Recovery Orientation - Survey Measures

IIIness self-management and goals were assessed through the IIIness Management and Recovery (IMR) Scales (Mueser \& Gingerich, 2005). Parallel forms of the scale are completed by the 
consumer and staff. Both IMR Scales have 15 items rated on a 5-point behaviorally anchored scale and include items such as progress toward goals, knowledge about mental illness, symptom distress, and coping. The IMR Scales have shown adequate internal consistency (Cronbach's alpha $\geq .70$ ), strong testretest correlations over a two week period (both versions, $r=.81, p<.001$ ), and have been correlated with other indices of functioning, symptoms, and recovery (Fardig, Lewander, Fredriksson, \& Melin, 2011; Hasson-Ohayon, Roe, \& Kravetz, 2008; Salyers, Godfrey, Mueser, \& Labriola, 2007).

Participant Activation. We assessed how active the consumer was in treatment with the mental health version of the brief Patient Activation Measure (Hibbard, Stockard, Mahoney, \& Tusler, 2004), a 13-item self-report scale designed to assess knowledge, skill, and confidence in health self-management. The scale is an interval, unidimensional, Guttman-like measure. Respondents endorse items (e.g., "I know what each of my prescribed medications do") on a scale from 1 ("disagree strongly") to 4 ("agree strongly"). The shortened 13 -item version of the scale has comparable reliability statistics and construct validity with the original 22-item version (Hibbard, Mahoney, Stockard, \& Tusler, 2005). The patient activation measure has been used successfully in samples of people with SMI (Alegria et al., 2008; Green et al., 2010; Salyers et al., 2009).

Hope was assessed using the 6-item Adult State Hope Scale (Snyder et al., 1996). A series of studies demonstrated the scale's internal consistency, high levels of convergent and discriminant validity, and sensitivity (Snyder et al., 1996). The scale has also been successfully used with people with SMI (McGrew, Johannesen, Griss, Born, \& Katuin, 2005).

Perceived Choice was assessed with a scale modeled after the Housing Choice Measure (Srebnik, Livingston, Gordon, \& King, 1995). The original scale includes 16 items. Respondents rate the degree of choice they have on issues related to housing (e.g., the place where you live, decoration and furnishing) using a 5-point scale from "not at all" to "completely my choice". The scale has adequate internal 
consistency, test-retest reliability, and interrater reliability (Srebnik et al., 1995). For the current study, we deleted items that were specific to a particular residence (e.g., decoration and furnishings) and added items to reflect choice in broader ACT services (e.g., whether or not to work).

Satisfaction with Services (SWS) scale is an 11-item consumer satisfaction checklist adapted from the Consumer Satisfaction Questionnaire (Larsen, Attkisson, Hargreaves, \& Nguyen, 1979). The SWS scale was designed specifically to be used with ACT consumers and has been used in several large-scale ACT studies (Bond, McDonel, Miller, \& Pensec, 1991; Bond et al., 1990; McGrew, Bond, Dietzen, McKasson, \& Miller, 1995). It has demonstrated adequate internal consistency (Cronbach's alpha $=.90)$ (Bond et al., 1990).

Optimism was assessed with the Life Orientation Test-Revised (Scheier, Carver, \& Bridges, 1994) and an adapted version of the Consumer Optimism Scale (Grusky, Tierney, \& Spanish, 1989). The Life Orientation Test-Revised is a 10-item measure that was designed to assess individual differences on personal optimism, and has adequate internal consistency, test-retest reliability, convergent validity, and divergent validity (Scheier et al., 1994). Both consumers and staff completed the personal optimism measure. Only staff completed the Consumer Optimism Scale, consisting of 16 items tapping the provider's expectations of consumers. The original 7-item Consumer Optimism Scale (Grusky et al., 1989) was expanded to include broader domains of recovery, and has been found to have strong internal consistency and test-retest reliability (Salyers, Tsai, et al., 2007).

\section{$\underline{\text { Recovery Orientation - Observer Rating Measures }}$}

Treatment plan ratings. We developed a checklist that assessors used to rate the recovery orientation of treatment plans on the basis of 1 ) evidence of consumer involvement in the development and review of plans, 2) the degree to which the plan reflects consumer goals and is individualized to the 
consumer's strengths and needs, and 3) the extent to which the progress notes show follow-through with interventions identified on the treatment plan. Ratings were made for 18 items on a 3-point scale indicating poor, moderate, or excellent quality of plans. Observers randomly selected 10 charts for review at each site. In the current study we found good internal consistency with Cronbach's alpha for the subscales ranging from .76 to .84 .

\section{Recovery Orientation - Staff and Consumer Rating Measures}

Treatment Control. We assessed the degree to which the control of treatment is in the hands of the consumer by gathering information on the presence of: involuntary outpatient commitment, guardianship, representative payeeship, lease-holder of the current residence, medication management strategies (e.g., independent self-administration vs. injection delivery), substance use monitoring (e.g., presence of urine/drug screens), family involvement, and contingent access to other desired services (e.g., help with competitive employment only if abstinent). Similar to Moser and Bond (2009), we asked team leaders to review the total roster of consumers and identify the number of consumers with each of the treatment mechanisms in place.

Visit diaries. Because diary methods are useful for tracking and examining daily interactions in social situations (Wheeler \& Reis, 1991), we used a form of Rochester Interaction Records (RIR) (Wheeler \& Nezlek, 1977) with staff and consumers to rate their interactions with one another. All available ACT staff were invited to keep 5 brief paper diaries for two days (10 total) to rate consumer interactions as they occurred. For each consumer interaction lasting at least 10 minutes, staff recorded the length of the interaction, treatment domain being addressed, and rated the interaction on five items: conveying hope, offering choice, fostering independence, encouraging risk/trying new things, and advancing consumer-defined goals. Ratings were made on a 5-point scale ranging from "not at all" to "very much". Consumers served by these staff during this two-day period were asked to complete 
parallel ratings for the same interaction and were given stamped envelopes to mail diaries to the research team. Staff and consumer forms had a research-assigned identification number to match them, but no identifying information of the staff or consumer involved.

Procedures

We approached sites to participate by contacting the team leader and his or her supervisor. We described the objective of the study-to identify best ways to measure recovery orientation on ACT teams-but did not explain the exemplar site selection. In some cases, we were asked to present to a larger management group. For sites agreeing to participate, we scheduled a total of six days of onsite visits with two researchers (one doctoral student in clinical psychology, paired with one of two clinical psychologists with expertise in ACT who were independent of the initial raters for the team selection). Visits were spread out over two to three months, and took place between July and December 2007. During our site visits, we reviewed assessments and treatment plans for 10 randomly selected consumers, conducted interviews, and distributed diaries. During most visits we also observed morning meetings and shadowed staff on visits to consumers when feasible. At the time of the initial visit, we distributed questionnaires to the ACT staff (Demographics, Optimism, and Consumer Optimism) and left additional questionnaires for the ACT program to deliver to all consumers (Demographics, Participant Activation, IMR ratings, Hope, Choice, Satisfaction, and Optimism). The consumer questionnaires included $\$ 5$ and a stamped, self-addressed return envelope. Each staff and consumer who participated in an interview received a $\$ 50$ gift card. Written informed consent was obtained for interviews, but we received a waiver of written informed consent for surveys by the Institutional Review Board. Each participating team also received $\$ 1500$ to offset costs associated with the time taken for site visits and other research-related activities. At the end of the study, we returned to teams to present and discuss findings [Author cite]. All procedures were approved by the Institutional Review Board at [University]. 


\section{Data Analysis}

We compared the two teams on all of the quantitative recovery orientation variables. We used chi square tests of significance for categorical data and independent sample $t$-tests for continuous data. We did not impute missing data, but analyzed only what data were present for that particular variable. We report on missing data as an important indicator of a method's utility. Because of the exploratory nature of this study, we did not adjust for multiple comparisons, which are procedures that can obscure potential findings particularly in exploratory contexts (Rothman, 1990). The intent was to see which types of measures may be most promising in differentiating teams, and we discuss any result as statistically significant at $p<.05$. We were also interested in feasibility of different methods of assessment and examined response rates and observations regarding different assessment methods.

\section{Results}

\section{Team Descriptions}

Teams had good to exemplary DACTS scores at the fidelity assessment prior to site visits: Team A scored 4.7 and Team B scored 4.1. Team A was a newer team, with a smaller caseload and was in an urban area.

For consumers completing surveys, the mean age was 42.3 years $(S D=9.9), 64.2 \%$ were female, 73.6\% were Caucasian, $83.0 \%$ were not married, and $41.5 \%$ had more than a high school degree. Teams did not differ significantly on consumer background characteristics. For staff, the mean age was 43.4 years $(S D=13.3), 63.2 \%$ were female, $84.2 \%$ were Caucasian, $42.1 \%$ were not married, all had at least a college degree and $31.6 \%$ had a graduate degree. Staff had been in their current positions a mean of 4.8 years $(S D=4.4)$ and had been in the mental health field a mean of 10.9 years $(S D=9.0)$. Teams differed significantly only on length of time in current position, with Team A having a shorter tenure (mean = 1.6; 
$S D=0.8$ years $)$, compared to 7.2 years $(S D=4.5), t=3.39, p<.001$. This roughly paralleled the length of time teams had been certified in (Team A for 1 year and Team B for 6 years). Medical records available for a subset of the caseload showed no significant differences in the rates of schizophrenia across teams $\left(57 \%\right.$ vs $\left.41 \%, X^{2}(1,103)=2.39\right)$ or in days hospitalized in the year prior $(M=10.6, S D=24.4$ vs $M=6.6$, $S D=26.6, t(83)=-0.65)$.

Surveys

Participation rates for consumer surveys were $47 \%$ (20 of 43 ) at Team $A$ and $45 \%$ (33 of 74 ) at Team B. Based on medical records, survey respondents were more likely to be female than those who did not respond to the survey $\left(66 \%\right.$ vs $\left.43 \%, X^{2}(1,100)=5.56, p<.05\right)$, but did not differ on race, education, marital status, diagnosis, or days hospitalized in the prior year. Staff survey response rates were $90 \%$ (8 of 9) at Team A and 83\% (10 of 12) at Team B. Internal consistency and the mean scores for surveys are shown in Table 1. All instruments except one (consumer-rated personal optimism) had good internal consistency at .75 or above. Overall, scores on the survey instruments did not differ significantly between teams, with the exception of staff-rated Consumer Optimism. Staff on Team A had significantly higher expectations regarding consumers $(t=2.90, p<.05)$.

\section{Observer Ratings}

Cronbach's alpha for the treatment plan reviews showed good internal consistency with subscales ranging from .76 to .84 . In terms of feasibility, we were able to complete 10 chart reviews at each site as planned. However, Team B had recently changed to electronic records and we were unable to access progress notes needed to rate one of the items. Another difficulty was the lack of specificity in our rating system, as one reviewer noted the need for "more clarification and objective criteria." Despite these difficulties, observer ratings of treatment plans showed substantial differences across 
teams (see Table 2). On two subscales (level of consumer involvement and treatment plan content), Team A was significantly higher than Team B. At the item level, Team A was significantly better on 10 of the 18 items. Although there were other areas in which Team A scored lower than Team B (regular reviews with consumers and having a specific time frame for goals), the differences were not statistically significant.

\section{Staff and Consumer Ratings}

Treatment control mechanisms, as reported by team leaders, are shown in Table 3. Team A reported significantly fewer proportions of consumers on four of the 10 control mechanisms assessed (representative payee, agency as lease holder, daily medication delivery, and regular family involvement). Neither team leader reported using guardianship, congregate living, or contingent access to services. We did, however, hear about examples of contingent access to some services at Team B during consumer and staff interviews.

Visit diaries had low response rates. Of 200 staff diaries distributed at the sites, 73 (37\%) were returned. Of those that staff completed, 58 (79\%) also had a consumer diary completed. The responses we did receive, however, revealed significant differences on all but one of the domains (conflict) for staff and consumers. Staff and consumers from Team A rated interactions as containing significantly greater levels of hope, choice, encouragement, independence, and trying new things. Consumers at Team A also rated significantly more advancement of consumer-defined goals on visits.

\section{Discussion}

We took a systematic approach to identify critical ingredients that would differentiate high and low recovery oriented ACT teams. We used multiple data sources and methods to identify the best assessment approaches that would capture differences and would be feasible to implement. In 
summary, a combination of observer, staff, and consumer ratings, supplemented with interviews may be the best approach for assessing recovery orientation on ACT teams (See Table 5).

The survey data were not very useful in our study in terms of discriminating levels of team recovery orientation. Although this method has relatively low burden and cost, only one scale showed a significant difference: Consumer Optimism. Staff at Team B had lower expectations for consumers. These low expectations were also supported in the interviews and observations [Author Cite]. Interestingly, staff with lower expectations had a longer tenure in their current positions. We have no way to know if expectations have changed over time; however, another study using the same scale found a correlation between length of time in current position and lower expectations for consumer success (Tsai \& Salyers, 2010).

Although staff differed in their perceptions of consumers, the consumers themselves did not differ in their own perceptions. That is, consumers on both teams rated themselves similarly in terms of being active in treatment and degree of illness self-management, hopefulness, optimism, and satisfaction. Consumer surveys had moderate to low participation rates, and it is possible that there was a sampling bias with the most satisfied, active participants at each site responding. Also, the surveys we chose may not reflect all of the critical principles underlying recovery (i.e., we may have measured the wrong constructs). However, we did measure choice, hope, self-management, and meaningful activity, which appear in other research (Noordsy et al., 2002; Resnick, Rosenheck, \& Lehman, 2004). Future work could be done to examine how consumers and staff are actually responding to survey questions. For example, cognitive interviews and think-aloud tasks while participants complete measures can be used to better understand the respondent's thinking process. Our interviews and observations, however, revealed that for some staff members, the spoken words 
about recovery and independence did not match observed behaviors. It is likely that surveys in general will not be adequate in assessing beliefs that may not be consistent with recovery ideals.

Observer ratings of treatment plans appear to present one opportunity for distinguishing teams, consistent with the movement for person-centered planning (Adams \& Grieder, 2005). Our assessment method, however, needs development, particularly in identifying objective anchors for ratings. We also found that for some items the concept did not lend itself to quality ratings per se, but was either present or absent rather than poor, moderate, or excellent (e.g., consumer present during treatment planning). We also noted that often a rating was the same across all charts at a site. For example, because of electronic records, one site had no consumer signatures on any of the plans. In this case, percentage of charts with or without a feature may not be very useful. One important caveat is that we were not blind to which team we were visiting when we made treatment plan ratings, and the ratings could be open to observer bias. However, Team B had a few items where they surpassed Team A (though not statistically significant), which suggests that our ratings were not totally biased. Still, future work should include raters blinded to condition. For example, site visitors could copy and de-identify plans and give them to blinded raters. In addition, inter-rater reliability should also be calculated on future measures.

Staff reports of treatment mechanisms appear to be another promising method to distinguish teams and were also low in assessment burden. In particular, representative payee, daily medication delivery, regular family involvement, and agency as lease-holder differentiated programs. This is a starting point, but frequency of use does not tell us how they use the mechanisms. There may be times when this level of team control is important (Frese, Stanley, Kress, \& Vogel-Scibilia, 2001). Even the highest recovery oriented team sometimes used these mechanisms. The difference, in our interviews and observations, was when and how they were used - as a last resort, after much discussion including 
consumer input, and with education about how mechanisms would be used and eventually discontinued [Author Cite].

The staff and consumer diaries were a good method of discriminating teams. Staff and consumer ratings significantly differed in the expected direction on all but 3 items. Given that these were observations by staff and consumers, rather than the researchers, we have greater confidence in the validity of the findings (i.e., not subject to our biases). However, the response rates were poor perhaps because of perceived burden or forgetting to complete them. In order to be useful, future work will require attention to increasing participation. Paper diaries can be augmented with some type of signaling device, such as pagers, preprogrammed wristwatches, or phone calls (Bolger, Davis, \& Rafaeli, 2003). Alternative methods including handheld electronic devices provide the benefits of signaling capabilities and the flexibility to tailor diaries to participants' schedules (Barrett \& Gill, 2001). Diary methods are frequently used in health care, with some evidence that response rates are better with electronic versus paper diaries (Stone, Shiffman, Schwartz, Broderick, \& Hufford, 2003).

Importantly, both ACT teams were above the usual cutpoint for what is considered adequate fidelity on the DACTS (score of 4.0). Although the more recovery-oriented team scored higher (by 0.6 points), we do not know how much of a difference is clinically meaningful. What may be more telling is that even in a high fidelity ACT setting, indicators of recovery orientation can still be present. This is consistent with other findings (Kidd et al., 2010; McGrew, Wilson, \& Bond, 2002; Moser \& Bond, 2009), and suggests that ACT fidelity itself is not in opposition with recovery principles. Indeed, a new fidelity scale has been developed to expand the DACTS to be more in line with recovery principles - the TMACT (Monroe-DeVita, Teague, \& Moser, 2011). In the early stages of validation, the relationship between the TMACT and recovery indices studied here are unknown, and represent a ripe area for future research. 
While we note strengths and weaknesses of different assessment methods, we also note that our study design was limited. First, we had a small sample - two ACT teams in one state - and may have been underpowered to find more subtle differences. In addition, we cannot adequately account for nested variables. Recovery orientation is part of the culture of a team of clinicians, serving particular consumers, who are embedded in an agency. In an ideal analysis, each of these levels should be accounted for statistically, and large numbers of teams would be needed for such analyses.

\section{Clinical Implications}

Nurses play a critical role on ACT teams (Cuddeback \& Shattell, 2010; Kane \& Blank, 2004; McGrew \& Bond, 1995; McGrew, Bond, Dietzen, \& Salyers, 1994) and can be leaders in shaping the recovery orientation of an ACT team. From a practice standpoint, treatment planning appears to be one key area for intervention focus. The high recovery oriented team had greater consumer involvement in treatment planning, and those treatment plans evidenced language supportive of consumer strengths and consumer-directed goals. An active role of consumers in treatment planning is a key element in promoting self-management of chronic conditions (Kemp, 2011) and is consistent with the recent emphasis on shared decision making in mental healthcare (Drake, Deegan, \& Rapp, 2010). In addition, there are models for treatment planning with useful strategies that could support recovery-oriented work in this area (Adams \& Grieder, 2005).

Beyond treatment planning, the area of treatment control is another clear candidate for psychiatric nurse involvement. Particularly salient for nursing is the finding that the high recoveryoriented team had far fewer consumers on daily medication monitoring. Similar to the documented role of community mental health nurses providing medication management (Wallace, O'Connell, \& Frisch, 2005), the ACT nurse plays a critical role in coordinating and administering medications, as well as providing medication education. By focusing greater resources on helping consumers to take 
medications independently, and integrating medications into recovery, nurses can take important steps in increasing the recovery orientation of their ACT teams.

Finally, the reflective diaries point to other steps nurses (as well as other members of the team) can take to assist in recovery. Conveying hope, offering choice, fostering independence, encouraging consumers, and working on consumer-directed goals were all areas of difference between the teams. Not surprisingly, these domains are similar to those that have been described by SAMHSA in their consensus work in defining recovery (SAMHSA, 2011), and appear in other work related to recovery support (Anthony, 2000; Deegan, 1996; Greenwood, Schaefer-McDonald, Winkel, \& Tsemberis, 2005; Noordsy et al., 2002; Resnick, Fontana, Lehman, \& Rosenheck, 2005).

\section{Conclusions}

We sought to identify, within one state, extremes of recovery orientation and used multiple methods to examine how teams differed. We used a crude rating system for selection and although ratings showed stability over time and consistency between raters, the ultimate validity of our exemplar criteria is untested. We did, however, find several notable differences between teams and some consistency across measurement approaches, strengthening confidence in the findings. In addition, because this was not a controlled study, there may be other factors distinguishing teams that impact results. For example, the teams differed in length of time as a team, team size, and rural/urban designation. Future studies of recovery orientation should include exemplars that represent these different aspects to help sort out critical factors that distinguish teams. Our next steps will be to distill and refine the most fruitful methods - observer ratings, staff ratings and reduced interviews - and apply them to other programs with more rigorous testing, for example, with raters blind to team selection. Ultimately, the relationship between recovery orientation and changes in consumer outcomes needs 
rigorous study. This study, however, was an important first step to begin systematically assessing recovery orientation. 
Table 1. Consumer and Staff Surveys

\begin{tabular}{|c|c|c|c|}
\hline \multirow[b]{2}{*}{ Consumer } & \multicolumn{3}{|c|}{$M(S D) /$ Significance } \\
\hline & $\frac{\text { Team A }}{(n=20)}$ & $\frac{\text { Team B }}{(n=33)}$ & t-test \\
\hline Participant Activation ${ }^{1}(\alpha=0.92)$ & $58.3(19.2)$ & $56.9(19.4)$ & 0.26 \\
\hline Satisfaction with Services ${ }^{2}(\alpha=0.92)$ & $2.6(0.4)$ & $2.4(0.5)$ & 1.80 \\
\hline $\operatorname{Hope}^{3}(\alpha=0.85)$ & $2.9(0.7)$ & $2.9(0.6)$ & 0.00 \\
\hline Choice $^{3} \quad(\alpha=0.89)$ & $4.0(0.8)$ & $4.0(0.8)$ & -0.08 \\
\hline Personal Optimism ${ }^{4}(\alpha=0.67)$ & $2.9(0.8)$ & $3.2(0.7)$ & -1.18 \\
\hline Self-Rated Illness Self-Management ${ }^{4}(\alpha=0.75)$ & $3.3(0.7)$ & $3.4(0.7)$ & -0.68 \\
\hline Clinician-Rated IIIness Self-Management ${ }^{4} \alpha=0.82$ ) & $3.0(0.7)$ & $3.2(0.6)$ & -1.38 \\
\hline Staff & $\frac{\text { Team A }}{(n=8)}$ & $\frac{\text { Team B }}{(n=10)}$ & $\underline{\text { t-test }}$ \\
\hline Personal Optimism ${ }^{4}(\alpha=0.76)$ & $4.0(0.5)$ & $4.1(0.5)$ & -0.53 \\
\hline Consumer Optimism ${ }^{4}(\alpha=0.83)$ & $3.4(0.3)$ & $3.0(0.4)$ & $2.90^{*}$ \\
\hline
\end{tabular}

Note: Consumer survey responses varied from $n=19-20$ at Team $A$ and $n=29-33$ at Team $B$

${ }^{1}$ Survey scores range from $0-100$.

${ }^{2}$ Survey scores range from $1-3$.

${ }^{3}$ Survey scores range from $1-4$.

${ }^{4}$ Survey scores range from $1-5$.

$* p<.05$ 
Table 2. Treatment Plan Reviews

\begin{tabular}{|c|c|c|c|}
\hline & \multicolumn{3}{|c|}{$M(S D) /$ Significance } \\
\hline & $\frac{\text { Team A }}{(n=10)}$ & $\frac{\text { Team B }}{(n=10)}$ & $\underline{\text { t-test }}$ \\
\hline Consumer's own words & $2.5(0.5)$ & $1.0(0.0)$ & $10.06 * *$ \\
\hline Consumer's signature on treatment plan & $2.4(0.8)$ & $1.0(0.0)$ & $5.39 * *$ \\
\hline Progress note $\&$ consumer involvement & $2.1(0.9)$ & $1.8(0.6)$ & 0.82 \\
\hline Consumer present when plan developed & $2.6(0.6)$ & $2.3(0.8)$ & 0.82 \\
\hline Regularly reviewed with consumer & $1.7(0.8)$ & $2.3(0.7)$ & -1.78 \\
\hline Evidence of Consumer Involvement Avg & $2.2(0.6)$ & $1.7(0.3)$ & $2.75 *$ \\
\hline Comprehensive consumer assessment & $2.2(0.4)$ & $1.0(0.0)$ & $4.02 * *$ \\
\hline Strength-based language & $1.8(0.6)$ & $1.0(0.0)$ & $4.00 * *$ \\
\hline Focus on how functioning is impacted & $2.5(0.5)$ & $1.3(0.5)$ & $5.21^{* *}$ \\
\hline Plan Content Avg & $2.2(0.5)$ & $1.1(0.2)$ & $6.44 * *$ \\
\hline "What consumers want to achieve" & $2.4(0.7)$ & $1.1(0.3)$ & $5.54 * *$ \\
\hline Objectives are small steps & $2.0(0.8)$ & $1.0(0.0)$ & $3.91 * *$ \\
\hline Goals have specific time frame & $1.1(0.3)$ & $1.2(0.4)$ & -0.54 \\
\hline Objectives have specific time frame & $1.7(0.5)$ & $2.1(0.9)$ & -1.38 \\
\hline Goals \& objectives are specific & $1.8(0.8)$ & $2.0(0.9)$ & -0.57 \\
\hline Goals \& objectives are realistic & $1.9(0.6)$ & $1.0(0.0)$ & $4.96^{* *}$ \\
\hline Goals \& objectives are measurable & $1.7(0.7)$ & $1.9(1.0)$ & -0.62 \\
\hline Goals \& objectives are individualized & $2.0(0.7)$ & $1.3(0.5)$ & $2.69 *$ \\
\hline Goals \& Objectives Avg & $1.8(0.5)$ & $1.5(0.4)$ & 1.93 \\
\hline Specific \& detailed intervention & $2.1(0.6)$ & $1.1(0.3)$ & $4.93 * *$ \\
\hline $\begin{array}{l}\text { Progress notes reflect goals, objectives \& } \\
\text { interventions }\end{array}$ & $2.0(0.5)$ & N/A & N/A \\
\hline Interventions Avg & $2.1(0.4)$ & N/A & N/A \\
\hline
\end{tabular}

Note: Ratings ranged from 1 = poor, 2 = moderate, 3 = excellent; Progress notes were not collected at Team $B$ due to electronic medical records filing systems.

$* \mathrm{p}<0.05$; $^{* *} \mathrm{p}<0.01$ 
Table 3. Control Mechanisms By Team Leader Report

\begin{tabular}{|c|c|c|c|}
\hline & $\begin{array}{l}\text { Team A } \\
(n=43)\end{array}$ & $\begin{array}{l}\underline{\text { Team B }} \\
(n=74)\end{array}$ & $\begin{array}{c}\text { Test of } \\
\text { Significance }\end{array}$ \\
\hline Involuntary outpatient commitment & $6(14.0 \%)$ & $20(27.0 \%)$ & $x^{2}=2.69$ \\
\hline Guardianship & $0(0.0 \%)$ & $0(0.0 \%)$ & $\mathrm{N} / \mathrm{A}$ \\
\hline ACT program is representative payee & $10(23.3 \%)$ & $31(41.9 \%)$ & $X^{2}=4.15^{*}$ \\
\hline $\begin{array}{l}\text { ACT program/agency is lease-holder of the } \\
\text { current residence }\end{array}$ & $2(4.7 \%)$ & $28(37.8 \%)$ & $X^{2}=15.71^{* *}$ \\
\hline Medication delivery daily with observation & $1(2.3 \%)$ & $29(39.2 \%)$ & $X^{2}=19.38^{* *}$ \\
\hline Injection medications & $11(25.6 \%)$ & $14(18.9 \%)$ & $x^{2}=0.72$ \\
\hline Regular (or random) drug screens & $0(0.0 \%)$ & $4(5.4 \%)$ & $\mathrm{N} / \mathrm{A}$ \\
\hline $\begin{array}{l}\text { Contingent access to other services (e.g., } \\
\text { housing if abstinent) }\end{array}$ & $0(0.0 \%)$ & $0(0.0 \%)$ & $\mathrm{N} / \mathrm{A}$ \\
\hline $\begin{array}{l}\text { Involvement with family on a regular basis } \\
\text { (e.g., weekly contact) }\end{array}$ & $5(11.6 \%)$ & $22(29.7 \%)$ & $x^{2}=5.02 *$ \\
\hline Congregate living situations & $0(0.0 \%)$ & $0(0.0 \%)$ & $\mathrm{N} / \mathrm{A}$ \\
\hline
\end{tabular}

Note: Chi-square values were not reported when small sample sizes violated the expected frequency cell size assumption.

$* p<0.05 ; * * p<0.01$ 
Table 4. Reflective Diaries

\begin{tabular}{lccc}
\hline & $\begin{array}{c}\text { Team A } \\
\text { Consumer n=25 } \\
\text { Staff n=29 }\end{array}$ & $\begin{array}{c}\text { Team B } \\
\text { Consumer n=23 } \\
\text { Staff n=44 }\end{array}$ & t-test \\
\hline Conveyed hope to the consumer & $4.6(0.6)$ & $3.6(1.1)$ & $3.85^{* *}$ \\
& $4.5(0.7)$ & $3.7(0.7)$ & $4.54^{* *}$ \\
\hline \multirow{2}{*}{ Offered the consumer choice } & $4.5(0.7)$ & $3.7(1.1)$ & $3.27^{* *}$ \\
\hline Encouraged the consumer to have more & $4.4(0.8)$ & $3.4(1.2)$ & $3.74^{* *}$ \\
realistic expectations & $4.4(0.9)$ & $3.0(1.3)$ & $4.57^{* *}$ \\
Fostered independence & $3.8(1.4)$ & $3.3(0.9)$ & $2.01^{*}$ \\
\hline \multirow{2}{*}{ Encouraged the consumer to try new things } & $4.4(0.8)$ & $3.7(1.3)$ & $2.45^{*}$ \\
& $4.4(0.8)$ & $3.8(1.0)$ & $2.96^{* *}$ \\
\hline \multirow{2}{*}{ In conflict with the consumer/staff } & $4.5(1.0)$ & $3.3(1.3)$ & $3.59^{* *}$ \\
\hline \multirow{2}{*}{ Advanced consumer-defined goals } & $3.8(1.2)$ & $3.2(1.2)$ & $2.06^{*}$ \\
\hline
\end{tabular}

Note: Ratings range from $1=$ not at all to $5=$ very much.

$* p<0.05 ; * * p<0.01$ 
Table 5. Summary of Assessment Approaches

\begin{tabular}{|c|c|c|c|c|}
\hline & $\begin{array}{c}\text { Overall } \\
\text { Response } \\
\text { Rate } \\
\end{array}$ & $\begin{array}{c}\text { Burden to } \\
\text { Respondents }\end{array}$ & $\begin{array}{l}\text { Burden to } \\
\text { Assessors }\end{array}$ & $\begin{array}{c}\text { Ability to } \\
\text { Differentiate }\end{array}$ \\
\hline \multicolumn{5}{|l|}{ Surveys } \\
\hline Consumer Surveys & $44 \%$ & low & low & \multirow{2}{*}{$\begin{array}{c}\text { Only Consumer } \\
\text { Optimism }\end{array}$} \\
\hline Staff Surveys & $88 \%$ & low & low & \\
\hline \multicolumn{4}{|l|}{ Observer Ratings } & \multirow{2}{*}{$\begin{array}{l}\text { Yes, but needs } \\
\text { refinement }\end{array}$} \\
\hline Treatment Plan Reviews & $100 \%$ & low & medium & \\
\hline \multicolumn{5}{|l|}{ Staff and Consumer Ratings } \\
\hline Treatment Control Mechanisms & $100 \%$ & medium & low & yes \\
\hline Staff Visit Diaries & $36.3 \%$ & low/medium & low & yes \\
\hline Consumer Visit Diaries & $66.9 \%$ & low/medium & low & yes \\
\hline \multicolumn{5}{|l|}{ Interviews } \\
\hline Consumer Interviews & $76.7 \%$ & low & high & yes \\
\hline Staff Interviews & $92.6 \%$ & medium & high & yes \\
\hline
\end{tabular}




\section{References}

Adams, N., \& Grieder, D. M. (2005). Treatment planning for person-centered care: the road to mental health and addiction recovery. Boston: Elsevier Academic Press.

Alegria, M., Polo, A., Gao, S., Santana, L., Rothstein, D., Jimenez, A., . . Normand, S. L. (2008). Evaluation of a patient activation and empowerment intervention in mental health care. Medical Care, 46(3), 247-256.

Angell, B., Mahoney, C. A., \& Martinez, N. I. (2006). Promoting treatment adherence in assertive community treatment. Social Services Review, 80(485-526).

Anthony, W. A. (2000). A recovery-oriented service system: setting some system level standards. Psychiatric Rehabilitation Journal, 24(2), 159 - 169.

Anthony, W. A. (2004). The principle of personhood: the field's transcendent principle. Psychiatric Rehabilitation Journal, 27(3), 205.

Anthony, W. A., Rogers, E. S., \& Farkas, M. (2003). Research on evidence-based practices: future directions in an era of recovery. Community Mental Health Journal, 39(2), 101-114.

Barrett, N. M., \& Gill, M. T. (2001). Web-based education in psychiatric rehabilitation. Psychiatric Rehabilitation Skills, 5(3), 548-571.

Becker, R. E., Meisler, N., Stormer, G., \& Brondino, M. J. (1999). Employment outcomes for clients with severe mental illness in a PACT model replication. Psychiatric Services, 50(1), 104-106.

Bedregal, L. E., O'Connell, M., \& Davidson, L. (2006). The Recovery Knowledge Inventory: assessment of mental health staff knowledge and attitudes about recovery. Psychiatr Rehabil J, 30(2), 96-103.

Bolger, N., Davis, A., \& Rafaeli, E. (2003). Diary methods: capturing life as it is lived. Annual Review of Psychology, 54(1), 579-616. 
Bond, G. R., Drake, R. E., Mueser, K. T., \& Latimer, E. (2001). Assertive community treatment for people with severe mental illness: Critical ingredients and impact on patients. Disease Management \& Health Outcomes, 9, 141-159.

Bond, G. R., McDonel, E. C., Miller, L. D., \& Pensec, M. (1991). Assertive community treatment and reference groups: An evaluation of their effectiveness for young adults with serious mental illness and substance abuse problems. . Psychosocial Rehabilitation Journal, 15(2), 31-43.

Bond, G. R., Witheridge, T. F., Dincin, J., Wasmer, D., Webb, J., \& De Graaf-Kaser, R. (1990). Assertive community treatment for frequent users of psychiatric hospitals in a large city: a controlled study. American Journal of Community Psychology, 18(6), 865-891.

Coldwell, C. M., \& Bender, W. S. (2007). The effectiveness of assertive community treatment for homeless populations with severe mental illness: a meta-analysis. American Journal of Psychiatry, 164(3), 393-399.

Cuddeback, G., \& Shattell, M. (2010). Nurses and assertive community treatment teams: a critical combination. Issues Ment Health Nurs, 31(11), 751-752.

Deegan, P. E. (1988). Recovery: The lived experience of rehabilitation. Psychosocial Rehabilitation Journal, 11(4), 12 - 19.

Deegan, P. E. (1996). Recovery and the conspiracy of hope. Paper presented at the Sixth Annual Mental Health Services Conference of Australia and New Zealand, Brisbane, Australia.

Dennis, D. L., \& Monahan, J. (1996). Coercion and aggressive community treatment: A new frontier in mental health law: Plenum Series in Social/Clinical Psychology.

Drake, R. E., Deegan, P. E., \& Rapp, C. (2010). The promise of shared decision making in mental health. Psychiatric Rehabilitation Journal, 34(1), 7-13.

Fardig, R., Lewander, T., Fredriksson, A., \& Melin, L. (2011). Evaluation of the Illness Management and Recovery Scale in schizophrenia and schizoaffective disorder. Schizophr Res, 132(2-3), 157-164. 
Frese, F. J., 3rd, Stanley, J., Kress, K., \& Vogel-Scibilia, S. (2001). Integrating evidence-based practices and the recovery model. Psychiatric Services, 52(11), 1462-1468.

Gomory, T. (2001). A critique of the effectiveness of assertive community treatment. Psychiatric Services, 52, 1394.

Green, C., Perrin, N., Polen, M., Leo, M., Hibbard, J., \& Tusler, M. (2010). Development of the Patient Activation Measure for Mental Health. Administration and Policy in Mental Health and Mental Health Services Research, 37(4), 327-333.

Greenwood, R. M., Schaefer-McDonald, N. J., Winkel, G., \& Tsemberis, S. (2005). Decreasing psychiatric symptoms by increasing choice in services for adults with histories of homelessness. American Journal of Community Psychology, 36, 223-238.

Grusky, O., Tierney, K., \& Spanish, M. (1989). Which community mental health services are most important? Administration and Policy in Mental Health, 17(1), 3-16.

Hasson-Ohayon, I., Roe, D., \& Kravetz, S. (2008). The psychometric properties of the illness management and recovery scale: Client and clinician versions. Psychiatry Research, 160(2), 228-235.

Hibbard, J. H., Mahoney, E. R., Stockard, J., \& Tusler, M. (2005). Development and testing of a short form of the patient activation measure. Health Services Research, 4O(6 Pt 1), 1918-1930.

Hibbard, J. H., Stockard, J., Mahoney, E. R., \& Tusler, M. (2004). Development of the Patient Activation Measure (PAM): conceptualizing and measuring activation in patients and consumers. Health Services Research, 39(4 Pt 1), 1005-1026.

Kane, C. F., \& Blank, M. B. (2004). NPACT: enhancing programs of assertive community treatment for the seriously mentally ill. Community Ment Health J, 40(6), 549-559.

Kemp, V. (2011). Use of 'chronic disease self-management strategies' in mental healthcare. Current Opinion In Psychiatry, 24(2), 144-148. 
Kidd, S., George, L., O’Connell, M., Sylvestre, J., Kirkpatrick, H., Browne, G., \& Thabane, L. (2010). Fidelity and recovery-orientation in assertive community treatment. Community Mental Health Journal, $46(4), 342-350$.

Larsen, D. L., Attkisson, C. C., Hargreaves, W. A., \& Nguyen, T. D. (1979). Assessment of client/patient satisfaction: Development of a general scale. Evaluation and Program Planning, 2, 197-207.

McGrew, J. H., \& Bond, G. R. (1995). Critical ingredients of assertive community treatment: Judgments of the experts. Journal of Mental Health Administration, 22, 113-125.

McGrew, J. H., Bond, G. R., Dietzen, L. L., McKasson, M., \& Miller, L. D. (1995). A multi-site study of client outcomes in assertive community treatment. Psychiatric Services, 46, 696-701.

McGrew, J. H., Bond, G. R., Dietzen, L. L., \& Salyers, M. P. (1994). Measuring the fidelity of implementation of a mental health program model. Journal of Consulting and Clinical Psychology, 62(4), 670-678.

McGrew, J. H., Johannesen, J. K., Griss, M. E., Born, D., \& Katuin, C. (2005). Performance-based funding of supported employment: A multi-site controlled trial. Journal of Vocational Rehabilitation, 23, 81-99.

McGrew, J. H., Wilson, R., \& Bond, G. R. (2002). An exploratory study of what clients like least about assertive community treatment. Psychiatric Services, 53, 761-763.

McHugo, G. J., Drake, R. E., Whitley, R., Bond, G. R., Campbell, K., Rapp, C. A., . . Finnerty, M. T. (2007). Fidelity outcomes in the national implementing evidence-based practices project. Psychiatric Services, 58(10), 1279-1284.

Mead, S., \& Copeland, M. (2000). What recovery means to us: Consumers' perspectives. Community Mental Health Journal, 36, 315-328. 
Monroe-DeVita, M., Teague, G. B., \& Moser, L. L. (2011). The TMACT: A new tool for measuring fidelity to assertive community treatment. Journal of the American Psychiatric Nurses Association, 17(1), 17-29.

Moser, L. L., \& Bond, G. R. (2009). Scope of Agency Control: Assertive Community Treatment Teams' Supervision of Consumers. Psychiatric Services, 60(7), 922-928.

Mueser, K. T., Bond, G. R., Drake, R. E., \& Resnick, S. G. (1998). Models of community care for severe mental illness: A review of research on case management. Schizophrenia Bulletin, 24, 37-74.

Mueser, K. T., \& Gingerich, S. (2005). Illness Management and Recovery (IMR) Scales. In T. CampbellOrde, J. Chamberlin, J. Carpenter \& H. S. Leff (Eds.), Measuring the Promise: A Compendium of Recovery Measures (Vol. II, pp. 124-132). Cambridge, MA: Evaluation Center @ Human Services Research Institute.

Neale, M. S., \& Rosenheck, R. A. (2000). Therapeutic limit setting in an assertive community treatment program. Psychiatric Services, 51, 499-505.

Noordsy, D., Torrey, W., Mueser, K., Mead, S., O'Keefe, C., \& Fox, L. (2002). Recovery from severe mental illness: An intrapersonal and functional outcome definition. International Review of Psychiatry, 14(4), 318-326.

O'Connell, M. J., Tondora, J., Evans, A., Croog, G., \& Davidson, L. (2005). From Rhetoric to Routine: Assessing Recovery-Oriented Practices in a State Mental Health and Addiction System. Psychiatric Rehabilitation Journal, 28, 378-386.

Phillips, S. D., Burns, B. J., Edgar, E. R., Mueser, K. T., Linkins, K. W., Rosenheck, R. A., . . McDonel Herr, E. C. (2001). Moving assertive community treatment into standard practice. Psychiatric Services, 52(6), 771-779. 
President's New Freedom Commission on Mental Health. (2003). Achieving the promise: Transforming mental health care in America. Final Report. DHHS Pub. No. SMA-03-3832. Rockville, MD: Substance Abuse and Mental Health Services Administration.

Rapp, C. A., \& Goscha, R. J. (2004). The principles of effective case management of mental health services. Psychiatric Rehabilitation Journal, 27.

Resnick, S. G., Fontana, A., Lehman, A. F., \& Rosenheck, R. A. (2005). An empirical conceptualization of the recovery orientation. Schizophrenia Research, 75(1), 119-128.

Resnick, S. G., Rosenheck, R. A., \& Lehman, A. F. (2004). An exploratory analysis of correlates of recovery. Psychiatric Services, 55(5), 540 - 547.

Ridgway, P. A. (Ed.). (2000). The Recovery Papers Volume 1: University of Kansas School of Social Welfare.

Rothman, K. J. (1990). No adjustments are needed for multiple comparisons. Epidemiology, 1(1), 43-46.

Salyers, M. P., Godfrey, J. L., Mueser, K. T., \& Labriola, S. (2007). Measuring illness management outcomes: a psychometric study of clinician and consumer rating scales for illness self management and recovery. Community Mental Health Journal, 43(5), 459-480.

Salyers, M. P., Matthias, M. S., Spann, C., Lydick, J., Rollins, A. L., \& Frankel, R. M. (2009). The role of patient activation in psychiatric visits. Psychiatric Services, 60(11), 1535-1539.

Salyers, M. P., Tsai, J., \& Stultz, T. A. (2007). Measuring recovery orientation in a hospital setting. Psychiatric Rehabilitation Journal, 31(2), 131-137.

Salyers, M. P., \& Tsemberis, S. (2007). ACT and recovery: integrating evidence-based practice and recovery orientation on assertive community treatment teams. Community Mental Health Journal, 43(6), 619-641. 
SAMHSA. (2011). SAMHSA announces a working definition of "recovery" from mental disorders and substance use disorders. SAMHSA News Release Retrieved 7/3/2012, from HTTP://WWW.SAMHSA.GOV/NEWSROOM/ADVISORIES/1112223420.ASPX

Scheier, M., Carver, C., \& Bridges, M. (1994). Distinguishing optimism from neuroticism (and trait anxiety, self-mastery, and self-esteem): A reevaluation of the Life Orientation Test. Journal of Personality and Social Psychology, 67, 1063-1063.

Snyder, C. R., Sympson, S. C., Ybasco, F. C., Borders, T. F., Babyak, M. A., \& Higgins, R. L. (1996). Development and validation of the State Hope Scale. Journal of Personality and Social Psychology, 70(2), 321 - 335.

Srebnik, D., Livingston, J., Gordon, L., \& King, D. (1995). Housing Choice and Community Success for Individuals with Serious and Persistent Mental Illness. Community Mental Health Journal, 31(2), $139-152$.

Stone, A., Shiffman, S., Schwartz, J., Broderick, J., \& Hufford, M. (2003). Patient compliance with paper and electronic diaries. Controlled Clinical Trials, 24(2), 182-199.

Teague, G. B., Bond, G. R., \& Drake, R. E. (1998). Program fidelity in assertive community treatment: development and use of a measure. American Journal of Orthopsychiatry, 68(2), 216-232.

Tsai, J., \& Salyers, M. P. (2010). Recovery orientation in hospital and community settings. Journal of Behavioral Health Services and Research, 37(3), 385-399.

Tschopp, M. K., Berven, N. L., \& Chan, F. (2011). Consumer perceptions of assertive community treatment interventions. Community Ment Health J, 47(4), 408-414.

Wallace, T., O'Connell, S., \& Frisch, S. R. (2005). What do nurses do when they take to the streets? An analysis of psychiatric and mental health nursing interventions in the community. Community Ment Health J, 41(4), 481-496. 
Weinstein, L. C., Henwood, B. F., Cody, J. W., Jordan, M., \& Lelar, R. (2011). Transforming assertive community treatment into an integrated care system: The role of nursing and primary care partnerships. Journal of the American Psychiatric Nurses Association 17, 64-71.

Wheeler, L., \& Nezlek, J. B. (1977). Sex differences in social participation. Journal of Personality \& Social Psychology, 35, 742-754.

Wheeler, L., \& Reis, H. T. (1991). Self-recording of everyday life events: Origins, types, and uses. Journal of Personality. Special Issue: Personality and daily experience, 59, 339-354.

Williamson, T. (2002). Ethics of assertive outreach (assertive community treatment teams). Current Opinion in Psychiatry, 15, 543 - 547. 